\title{
II. Ueber Protozoenbefunde im Ovarial- und Uteruscarcinom
}

\author{
Von \\ Privatdocent Dr. VITALIS MULLER \\ in St. Petersburg. \\ (Hierzu 10 Albildungen.)
}

Ungeachtet dessen, dass Untersuchungen ïber Protozoa an gehärteten Präparaten augenscheinlich wenig Wert haben, wie solches ja die ganze umfangreiche Litteratur iber die Carcinomparasiten beweist, entschliesse ich mich dennoch Nachfolgendes der Oeffentlichkeit zu ïbergeben. Der Streit iiber den Carcinomparasiten kann endgiltig zweifellos nur durch Lntersuchungen am frischen lebenden Object entschieden werden und sind ja hierin ron mehreren Seiten bereits rühmliche Anfänge gemacht. Doch glaube ich andererseits, dass Studien am gehärteten Object uns manche Winke geben können, wohin wir hauptsächlich unser Augenmerk zu richten haben werden, wenn der Schwerpunkt unserer Arbeiten uber die cellulären Vorginge im Carcinom gan\% anf's frische Gewebe iabertragen sein wird. Nachfolgende Zeilen sollen darum nur als Vorstudium gelten.

1. '/uerst erlaube ich mir über einen Fall von Carcinoma ovarii \%u berichten.

Die betreffende Kranke war 46 Jahre alt, hatte 8 mal geboren. Nach der letzten Geburt, zehn Jahre vor ihrem Eintritt in ursere Anstalt, hatte ihr Haus. arzt eine chronische Metritis c:instatiert und sie lange Zeit daran behandelt.

In Mai 189:3 bemerkt die Kranke, dass ihr Iauch an Unfang zunimmt. I)er Hausaryt constatiert einen langsam wachsender Ovarialtumor. WWecks Entfernung dieses 'l'unors kommt die Kranke am 7. 9. 1893 aut dic grnaekologische Abteilung des Herrn l'rof. lirassowsky. Inden ich den genauen Status praesens bei der Aufnalıme übergehe, erlaube ich nir nur zı notieren, dass am 11i. 9. Prof. Krassowsky olme jegliche Schwierigkeiten einen Tumor des re:hten lierstockes ron $1400 \mathrm{~g}$ und einen solchen des linken von $100 \mathrm{~g}$ per ('oeliotoniom entfernte. Reide 'lumuren waren langgestielt. Ausserdem constatierte die in den Bauchraum eingeführte Hand einen dritten platten, høckerigen, utwa $7-8$ (puerfinger breiten Tumor. Welcher der Aorta unbeweglich aufsass und bis zur linken Niere reichte. Dieser 'l'umor wurde in situ gelassen. Glatter Verlauf nach der Operation. Als die Kranke am 1:3. 10. die Anstalt verliess. wurde constatiert. dass die nicht entfernte Geschwulst sich etwas vergrössert hatte, dass die Lymphdrüsen in der rechten Fossa supraclaricularis etwas geschwollen waren und man über dem rechten Poupartschen Bande einen unbeweglichen Tumor ron Hïhnereicrrosse palpieren konnte. Auf (irund eines solchen klinischen Bildes konnte es wohl keinem Zweifel unterliegen, dass die Kranke an einer höchst malignen Neubildung litt.

Makroskopisch hatten dic entfernten 'lumoren alle Eigenschaften eines Markschwamnes.

Fïr die mikroskopische Untersuchung wurden kleine Geurebsstückchen unnittelbar nach der ()peration aus beiden Tumoren herauscreschnitten, in Alcohol absul. fixiert und nach der ron Heidenhain ") angegebenen Mechode in Paraffin

1) Heidenhaill. Ueber Kern und Protuplasma. Leip\%ig 1892.

Sonatsschrift für (ielurtshulfe und Gyaakologie. Bd. I. Heft 6. 
eingebettet. Die Mlikrotomschnitte wurden mit destilliertem Masser auf den Objectträger geklebt. Fürbung mit Ehrlich's T'riacidlüsung, nach Russel's Methode und der Weigert'schen Bacterientarbung.

Mikroskopisch bestanden die 'I'umoren fast ausschliesslich aus Epithelzellnestern, welche in kümmerlich entwickeltem faserigen Bindegewebe eingebettet waren. Die Epithelzellen lagen nicht fest aneinander. Untersuchte man ein Prăparat unmittelbar nach dessen Einschliessung in Xylulbalsam und drückte etwas aufs Deckglas, so konnte jede Epithelzelle in beliebiger Richtung verschoben werden. Bei weiterer Untersuchung erwiesen sich die meisten Zellen als einkernige; zuweilen konnte man übrigene auch zwei- und dreikernige antreffen. In jedem Kern sab man ein oder mehrere, manchmal recht viele Gebilde, welche Kernkörperchen sehr ̋̆hnelten. Zuweilen wurden solche Korper auch im Plasma angetroffen. Meistens rund und von verschiedener Grơsie, hatten sie ausnabmsweise ein unregelmăssiges resp. amoeboides Aussehen. Weiter sah man Zellen mit Vacuolen, welche ebensolche Korper entbiolten, nur das let\%tere hier mit einem kaum homogenen Plasma umgeben waren. An der Peripherie der Vacuole konnte man zuweilen Kernreste nachweisen; in den meisten racuolisierten Zellen war jedoch von einen Kern keine Spur. Findlich sah man solche mit homogenem

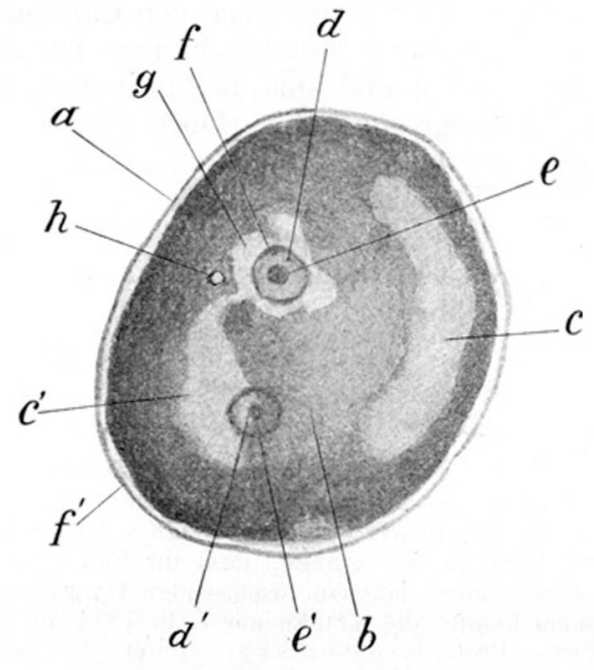

Fig. 1.

Plasnı umgebene Kurper auch frei $z$ wischen den anderen \%ellen liegen: sebr oft waren \%wei solche Kürper in einem Stück Plasma enthalten. Man muss annehmen, dass letztere Gobildo anfinglich wohl auch in Kollonvacuolon ontbalten waren und erst nach vollstiindigem Zerfall der \%olle frei geworden sind. Gewobnlich sind sie bedeutend gröser als solcho, welche noch in augenscheinlich intacten Kernen sich botinden. Ueherbaupt war es nicbt schwer, sich zu überzeugen, dass, je grösser die Kernkorperchen, umsoweniger deutlicb die Kernumrisse waren, umso ausgesprochener die Vacuolisation der Zelle: mit anderen Worten, der Kernschwund, die Vacuolenbildung und der endliche Verfall der Zellen gehen gleichzeitig mit dem $W$ achsthum des quasi Kernkörperchens einher. Die meisten dieser Kurper farben sich mit der Fbrlich'schen Triacidlosung rot; einige schimmern gleichzeitig sowohl rot, als auch blau: ausnahmsweise konnte man in einem roten Grundstoff kleine blaue Kügelchen sehen. Aehnlich farben sich diese Gebilde auch nach der Russel'schen Methode. Nur trifft man hier zuweilen solche, wo in einen blauen Grundstoff rote Kügelchen eingeschlossen sind. Figuren, welche als Teilungserscheinungen dieser Gebilde zu deuten wären, babe ich nicht beobachtet. Eeber die Natur dieser Kürper kann ich nichts aussagen. Man kơnnte an Parasiten oder irgend einen Degenerationsvorgang der Zelle denken. Um solch einem Gebilde den Namen eines Parasiten zu geben, müsste 
man ausser Plasma einen deutlichen Kern nachweisen. Dieses ist mir aber kein Mal gelungen. Diejenigen kleinen Partikelchen, welche in manchen von ihnen enthalten sind und nach Ehrlich blau, nach Russel sich rot fürbon, als Kerne zu betrachten, halte ich für zu problematisch. Erklărt man sicb diese Gebilde da fregen als Degeneriationserscheinungen, so ist es wiederum nicht ganz klar, mit was fuir einer Art von Degeneration wir es bior zi thun haben. Darum ist es einfacher zu sagen, ich wisste nicht, was das für Korper seien.

Ausser den eben beschriebenen habe ich noch Korper von ganz anderem Aussehen aufgefunden. Das waren grosse Gebilde, welche allo extracellular sich befanden. Nach den Zeichnungen Fig. 1-4 kann man sicb unschwer überzeugon, dass es Cysten mit oder obne Inhalt sind. Auf Fig. 1 ist ein Korper abgebildet, welcher nach Weigert intensiv rơtlich-violett getärbt war. Man kann roll-

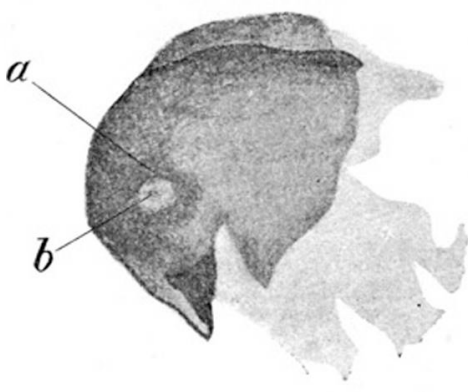

Fig. 2.

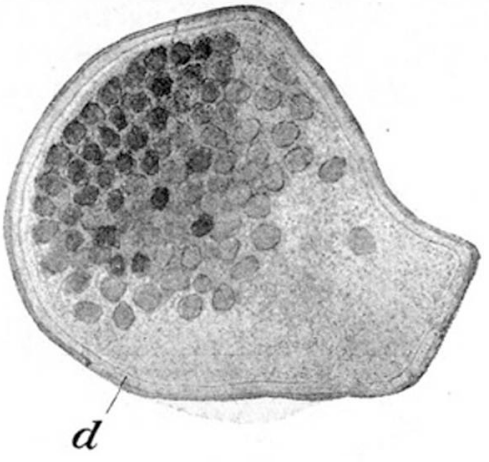

Fig. 3.

kommen deutlich eine Membran a und einen Inbalt b unterscheiden; letzterer ist, wobl infolge der Reaction. etwas von der Membran abgehuben. Der Inhalt ist nicht rollkommen gleichmassier gefarbt. sondern man kann bier irgend welche bellere Streifen $c^{\prime}$ und zwei helle Blasen ${ }^{1}$ ) (dd') mit deutlichem dunkeln Contur ( $\left.f f^{\prime}\right)$ und einem kleinen dunkeln Körperchen in der Mitte (ee'l unterscheiden. Diese Blasen sind nun nichts anderes, als sogenannte bliischenformige Kerne, deren Bestandteile - Membran, heller Inhalt und Binnenkürper - sich vollkommon deutlich darstellen, Der obere Kern (d) ist von einen hellen unregelmässigen Hof' (g) umgeben. Bei (h) sicht man noch irgend ein helles, glinzendes Korpercben. Aut Fig. 2 ist eine (vielleicht mit dem IIkrotom) zerrissene Cysto zu seben, welche auf dem nach $R$ ussel behandelicn l'riiparate blau gefarbe war. Innerbalh der Membran (a), an deren linkem Runde, befindet sich ein etwar dunkler geflirbtes, feingekorntes protoplasmatisches (iebilde (b) nit verschwommenen Lmrissen. In diesem Plasma ist eben ein solches I3lasschen mit einem Binnenkörper. Wie aut Fig. 1 \%u sehen.

Diese Priparare habe ich dem P'rivatdoconten für Zoulogie. friiher an der Heidolberger, gegenwiirtig an ler sit. l'etersburger Universităt, I)r. Sch ewiak off und dem Assistenten am botanischen Institut der milithrmedicinischen Akademie, I)r. W ahrlich. demonstriert. Beide anerkannten die beschriebenen Gebilde als blischent'ormige Kerno, welche bekanntlich fïr viole niedere l'rotw\%oa charakteristisch sind. Auf (irund les Vorhandenseins solcher Kerne mit bestimmtem. rollkommen deutlichem Bau, kann man nun gan\% positiv bebaupten, dass auf Fig. 1 und 2 unzweifelhafte Protozoa rus einem carcinomatissen 'l'umor abgebildet. sind.

Aut I Ig. 3 ist cine weitere Cyste. oftentar im Durchschnitt, abgebildet. Dic: Membran resp. Kapsel (a) ist doppelschichtig, erliinzend, nicht gefarht: der Inhalt besteht aus einer Monge irgend welcher kleinen rundon Kirpcrchen von untercinander gleicher firosse, welche nacb Weigert rothraun gefurbt waron.

1) Als ich iiber diesen Fall im Wratsch No. 71895 bericbtete, hatte ich die \%weite Blase (d') noch nicht gesehen. 
Was dieses für kïrper sind, weiss ich nicht. Vielleicht konnten es Sporen sein. Nach ihrer Grösse und ihrem Verhăltnisse zum ubrigen Carcinomgewebe. unterscheidet sich diese Cyste nicht ron denjenigen, welche in Fig. 1 und 2 abgebildet sind. Ausser dieser habe ich noch mehrere audere Cysten gesehen, durch deren Membran ebensolche Korperchen, freilich nicht so doutlich, durchscbimmerten.

Auf Fig. 4 sieht man eine (nach Russel war sie blau gefarbt) weitere Cyste mit regelmüssigem doppelten Contur. In der Wand dieser (yste kann man bei gerisser Finstellung des Objectirs drei eiformige Oeffnungen (a) constatieren. Bei Aenderung der Einstellung siebt man noch zwei weitere, hier nicht gezeichnete Oeffinungen in der rechten Hulfite der Cyste. Dieses Gebilde kann man sich ohne Mühe erklaren, indem man es für eine leere parasitäre Cyste hălt und annimmt. dass die Parasiten durch die fünf Oeffinungen die Cyste verlassen haben. Ich babe noch mehrere zerrissene und auf verschiedene Weise zusammengefaltete Membranen, derjenigen auf Fig. 2 ausserst abnlich, gesehen. Zuweilen

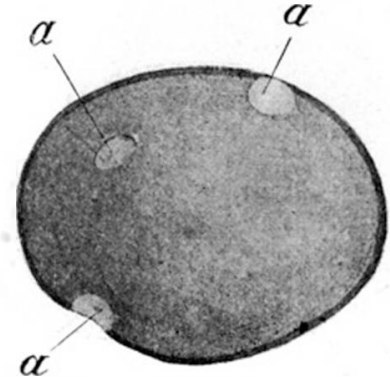

Fig. 4.

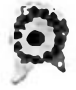

Fig. 5.

lagen neben denselben ebensolche kleine glänzende Korperchen, wic in Fig. 1 b. Weiter habe ich noch einige intacte Cysten gefunden, deren Inhalt so intensiv gefarbt war, dass man gar keine structur darin erkennen konnte.

Ich orlaube mir $2 \mathrm{u}$ wiederholen, dass alle beschriebenen Gebilde extracellulïr und ofter vollkommen frei lagen. Untersuchte man das bezúgliche Priiparat unmittelbar nacb dessen Einschliessung in Xylolbalsam, so konnte man seben, wie einige von den intacten Cysten sich um irgend eine Ase drehten; beim leisesten Druck auf's Deckglas wurden sie in beliebiger Richtung dislociert.

Ich habe folglich Cysten gesehen, erstens mit einem Inhalt von höchst unbestimmter Art, wie Fig. 3, oder auch ohne eines solchen, Fig. 4, zweitens mit einem Inhalt, welcher nur auf eine Weise erklärt werden kann, indem man ilin als Parasiten anerkennt, da ein Kern von ganz bestimmtem Baue vorhanden war. Solcher Cysten habe ich im ganzen zwei, eben diejenigen, welche hier abgebildet sind, gefunden. In den ubrigen Cysten und leeren Saicken waren keine Andeutungen ron einem ähnlichen Kern. Was aber ihre Grösse, Farbung und Beziehung zum übrigen Gewebe anbelangt, so unterscheiden sie sich nicht von den zwei ersten. Darum glaube ich nicht fehlzugehen, wenn ich alle diese Gebilde als zusammengehörend auffasse: es sind eben nur verschiedene Varie. täten oder Entwicklungsphasen desselben parasitären Organismus. Vielleicht werden künftige Untersuchungen mit vollkommneren Methoden die Bedeutung aller dieser Gebilde aufklären.

Die geschilderten Cysten stammen alle nur aus einem Gewebsstiickchen; in den übrigen habe ich nichts äbnliches auffinden können.

Dergleichen extracellular gelegene grosse Parasitencysten in krebsigen Neubildungen sind bereits mehrere Mal beschrieben, wie 
z. B. ron Albarron ${ }^{1}$ ) und sogar von Gegnern der Carcinomprotozoen wie Noeggerath ${ }^{2}$ ). Von einem bläschenförmigen Kern ist aber bei diesen Autoren nirgends die Rede. Fs giebt sogar Autoren, wie Korotneff ${ }^{3}$ ), welche ausdrücklich betonen, der Kern des Carcinomparasiten sei kein bläschenförmiger. Ich freue mich darum ganz besonders, in Lawtschenko ${ }^{4}$ ), was diesen Punkt anbelangt, einen Bundesgenossen gefunden $\mathrm{zu}$ haben. In seiner letzten Arbeit ïber den uns interressierenden Gegenstand schildert er die Kerne der von ihm gesehenen Parasiten als bläschenförmige. In allen ubrigen Details stimmen freilich meine und Lawtschenko's Schilderungen nichts weniger als iuberein.

II. Es sei mir erlaubt hier noch einige Befunde $z u$ verzeichnen, die ich in Uteruscarcinomen erhoben habe, als Ergänzung zu denjenigen, uber welche ich andern (Ortes ${ }^{5}$ ) bereits berichtet habe.

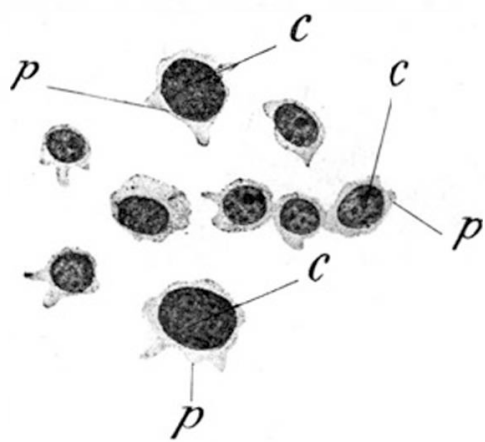

Fig. 6.

Die betr. Gewebspartikel wurden mir Dank der Liebenswürdigkeit des Herrn Dr. Wasten aus dessen gynäkologischer Abteilung ( $\mathrm{Ob}$ uchow'sches Krankenhaus), in $97 \%$ Alkohol eingelegt, zur Untersuchung übergeben. Sie staminten von einem inoperablen Partiocarcinom und waren bei einer Auskratzung gewonnen. Die Einbettung wurde wie oben ausgefuhrt. Fürbung der Schnitte nach Russel und Weigert. Bei der mikroskopischen Úntersuchung wurde ein Carcinoma cylindrocellulare solidum mit bäufigen '/erfallsherden constatiert. In der Năhe solcher /erfallsherde fanden sich zuweilen massenbaft Einschlusse, die sich obne Schwierigkeit als Necrophagismus (Podw yssotzky) erklaren liessen. Nachdem ich eine ganze Menge von Schnitten, aus verschiedenen Gewebsstückchen stammend, vergeblich nach Parasiten durchmustert, fielen mir plotzlich mehrere Gruppen besonderer ('iebilde auf, von denen einige auf Fig. 5 und 6 bei stirkorer und Fig. 7 béi schwiicherer Vergrosserung abgebildet sind. Sie lagen alle extracellulär. Bei genauerem Zusehen überzeugt man sich, dass ein jedes von ihnen aus einem peripherischen (p) und einem centralen (c) Anteile besteht. I)ie Úmrisse des sebr zart aussehender. peripherischen Anteils sind meistens unregelmíssig, zuweilen wie zusammengefaltet. Nach Russel färbt er sich schwach blau.

Der centrale Teil bez. der Inhalt stellt sich mehr weniger rund dar und auf den ersten Blick rot gefirbt. Bei stärkeren Vergrösserungen sieht man aber

1) Albarron. Les tumeurs de la vessie. Paris 1892.

2) Noeggerath. Beiträge zur Structur und Entwickelung des Carcinoms. Wiesbaden 1892 .

8) Korotneff. Ueber den parasitaren Charakter der Krebsgeschwulst. Kiew 1893 (russisch). (russisct:).

4) Lawtschenko. Ueber Sporozoa in malignen Tumoren. Kiew 1894

5) Archiv f. Gynaekol. Bd. XILIII. R. 2. 
deutlich, dass er aus einer Anhäufung von grösseren und kleineren roten Krormern in irgend einem Medium besteht. Der Durchmesser dieser Gebilde schwankte gewobnlich zwischen 2-3 $\mu$, höchstens $4 \mu$. Die bez:Iglichen Präparate habe ich den Herren Prof. Grobi (Botanik), Privatdocenten Schewiak off ('Loologie), Dr. $\mathrm{W}$ ahrlich (Botanik) demonstriert und alle drei definierten den blauen peripheren Anteil als Hiille (resp. Kapsel) und den centralen als vermutlichen Parasiten. Einen ganz tadellosen Kern ist es mir leider nichi gelungen in letzterem nachzuweisen. Zuweilen batte es wohl den Anschein, als befinde sich ein blüschenformiger Kern im Centrum des Parasiten (Fig. 5. k.); doch waren das nur kusserst spärliche Exemplare und trat der Bau des vermutlichen Kerns durchaus nicht so klar hervor, wie im oben beschriebenen Orarialcarcinom.

Diese Gebilde lagen nun immer gruppenweise 10-100 zusammen, ausserst selten konnte man einzelne Exemplare antreffen. Bald lagen sie zwischen Epithelzellen, bald zwischen Bindegerwebe; ausnahmsweise auch in Zerfallberden. Sehr oft befanden sich solche Gruppen, im Fpithelgewebe liegend, hart an der Grenze eines Zerfallberdes. Finmal fand ich sie in einen Gerass; doch konnte ich nicht entscheiden, ob es eine Vene oder ein Lymphgefass war, - rote Blutkörperchen waren darin allerdings nicht enthalten. Zuweilen konnte man in

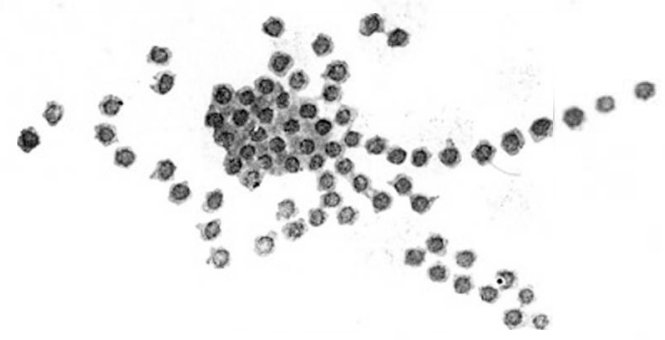

Fig. 7.

manchen von diesen Gruppen wie eine Neigung zu Kettenbildung constatieren (Fig. 7). Letztere Gruppe lag gerade auf der Grenze von Epithel und Bindegervebe.

Nicht immer hatten die Gebilde das eben beschriebene Ausseben. Einigemal habe ich kleine Gruppen angetrofien, wo die Hülle (Fig. 8 p) nicht so zart

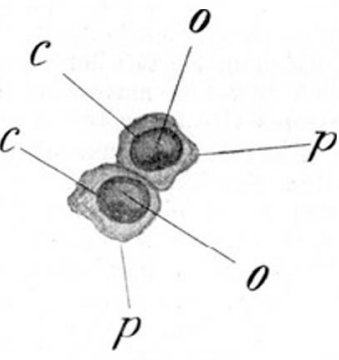

Fig. 8. aussah und husserst schwach tingiert war. Auch der Inhalt (c) hatte hier wenig Farbe angenommen und war nur dessen Peripherie rot gefärbt: das Centrum (o) blieb aber ungefarbt und sah wio eine Vacuole aus. Man kornnte sagen, der Inbalt nehme umsoneniger Farbstoff auf, je fester die Hiille ist.

In den meisten Gruppen waren die Gebilde untor einander von gloichor Grüsse: nur sehr selten von rerscbiedener.

In den nach Weigert behandelten Präpa. raten war die Hülle stark violett, der kornige Inbalt dagegen schwach tingiert.

Ausser den eben beschricbenen fanden sich in diesen Carcinom norh Gruppen von ganz anders aussehenden Gebilden, deren mebrere in Fig. 9 gezeichnet sind. Es sind das langliche Korper, welche aus einem grosseren und einem kleineren, durch einen schmalen Hals verbundene Toile bestehen. Sie färben sich nach Russel rotlichbraun und sind ziemlich grobgekornt. Ihre Lange betrügt 9 u. Nur einmal konnte ich etwas, das wie ein bläschenförmiger Kern (Fig. 9 k) aussah, auffinden; sonst war von deutlichen Kernen keine Spur. Nach Weigert wurden die Korner intensiv dunkelviolett gefarbt und konnte man auch an der Peripherie des Gebildes einen einfache dunkel gefurbte Contur nachweisen. Das Verhaltnis dieser Gebilde zum übrigen Gewebe war genau dasselbe, wie der zuerst beschriebenen. 
Gruppen von solchen Gebilden, sowohl der ersten, wie der $\mathbf{z}$ weiten Gattung, habc ich nur in vier Serienschnitten aus einem Gewebspartikelchen des betr. Portiocarcinoms aufgefunden. In allen übrigen Schnitten habe ich umsonst nach solchen gesucht.

Wie sind nun diese Gebilde zu deuten: als Degenerationserscheinungen, oder als Proto\%oa? Beim Versuch, die S'ache auf die erste Art zu erklïren, weiss ich wirklich nicht, welche Degenerationsvorgänge, welche Gewebselemente ich herbeiziehen soll. Die Gebilde fanden sich sowohl in Epithel, wie Bindegewebe, wie in Yerfallsherden, als auch einmal in einem Gefäss. Man könnte folglich an degenerierte Leukocyten oder rote Blutkörperchen denken. Aus der Beschreibung und den Abbildungen geht es aber andererseits dentlich genug hervor, dass unsere Gebilde sowohl der Grösse, wie dem Bau nach absolut keine, nicht einmal entfernte tehnlichkeit mit diesen Blutbestandteilen haben. Auch konnte ich nirgends Andeutungen von etwaigen Uebergangsbildern ron den einen zu den andern auffinden. Es fehlen somit jegliche Anhaltspunkte, die Gebilde als Degenerationsworgang zu deuten. Iagegen meinten die oben genannten Herrn Botaniker und Zoologen, dass sie sich diese

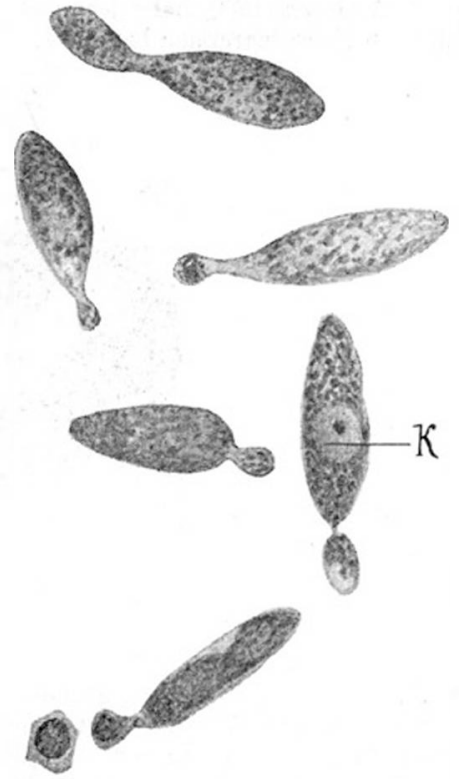

Fig. 9. (iebilde als Protozoa sehr wohl denken könnten, ungeachtet, dass ein Kern mit Sicherheit nicht nachgewiesen war. Dieser Umstand mïsste als Folge der Fixirungsmethode erklärt werden. Es unterliegt ïberhaupt keinem Zweifel, dass die äussere Form der beschriebenen Gebilde durch die Präparationsmethode wohl bedeutend verändert war und wïrden sie eventuell im frischen lebenden Zustand ein etwas anderes Aussehen haben. Ich bin mir folglich sehr wohl bewusst, dass ich einen strikten Beweis fur die parasitäre Natur der beschriebenen Gebilde schuldig geblieben bin; und ist es das Fehlen jeglicher Anhaltspunkte, etwaige Degenerationsvorgänge \%ur Erklärung herbeizuziehen, was mich bestimmt hier Proto\%oa zu vermuten. Angenommen, es seien Protozoa, so entsteht eine weitere Frage, wie sich beide beschriebenen Arten \%u einander verhalten: sind es verschiedene Parasiten, sind es Modificationen ein und derselben Art? Beide waren nur in denselben vier Sclinitten vorhanden, beide kamen gruppenweise vor, in beiden war das Plasma grobgekörnt und rot gefärbt, in beiden der Kern höchst undeutlich. Diese vier Punkte bestimmen mich \%u vermuten, dass es sich wohl, trot\% ihres verschiedenen Aussehens, nur um Evolutionsformen eines Parasiten handelt. Die erste Art habe ich in noch zwei weiteren 
Fällen ron inoperablen Portiokrebs igynäkologische $A$ bteilung des Herrn I)r. Woster) und in einem Falle von Corpuscarcinom (gynäkologische Abteilung des Herrn Prof. Krassowsky) gesehen. In beiden Portiokrebsen habe ich aber trotz vieler Mühe nur je cine solche Gruppe auffinden können; im Corpuscarcinom fand ich vier Gruppen, jedoch alle in einem Schnitt. Das ist alles, was ich iber diese sonderbaren (jebilde angeben kann.

Andererseits ${ }^{1}$ ) habe icb noch über intracellulare Organismen in einem Falle von Uteruscarcinom berichtet. Ist es nun uioglich, die hier beschriebenen extra-

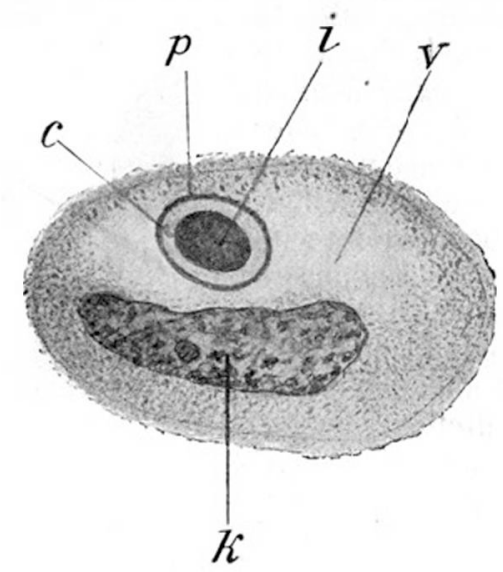

Fig. 10.

cellularen mit jenen in genetiscben /usammenbang zu bringen? Ich muss gesteben, dass den čusseren Aussehen nach auf fixierten Präparaten solches wohl schwer möglich ist. In jenem Falle konnte ich zudem keine einzige extracellulare Gruppe auffinden. Nach reche langem Studium der Präparate beider Fulle habe ich aber doch einen schwachen Anhaltspunkt aufgefunden. Auf Fig. 10 ist ein Einschluss aus dem hier beschriebenen Falle von Portiokrebs abgebildet. In einer Epithelzellenvacuole $(v)$ befindet sich ein Gebilde (p). Der Zellkern (k) ist etwas platt gedrückt. Das eingeschlossene Gebilde besteht aus einer recht dicken Kapsel (c) und einem nach $R$ ussel blau gefärbten Inhalt (i). Eine parasitäre Cyste kann hier bestimmt nicht ausgeschlossen werden. Solche Einschlüsse habe ich nun ziemlich biufig, sowobl in der Nahe der beschriebenen Gruppen, als auch in jenem Falle nit amoebenartigen Finschliissen gefunden. Dies ist der einzige geringe Fingerzeig, den icb für die Zusammengebørigkeit der von mir boschriebenen intra- und extracellularen Formen beibringen kann. Von einem Beweis ist allerdings keine Rede.

Leberhaupt sehe ich sehr gut ein, dass alles, was ich hier uber die extracellularen Parasiten im Uteruscarcinom gesagt habe, sich vielmehr auf Vermutungen, als positive Beweise stittrt. Dessen ungeachtet erlaubte ich mir die Sache zu veröffentlichen, da ich bei keinem von den Autoren, welche gegenwärtig iiber Carcinomparasitismus arbeiten, etwas über ähnliche Parasitengruppen gefunden habe und hoffe, damit das Material der heute so viel umstrittenen Gebilde etwas zu vervollständigen.

Zuletzt sei es mir erlaubt, meinen innigsten Iank auszusprechen den Herren Prof. Krassowsky und Dr. Waster fur Ceberlassung

1) I. c. 
des Materials und den Herren Prof. Gobi, Privatdocent Schewia$\mathrm{k}$ off und Dr. Wahrlich fiur ihre liebenswiurdigen Ratschläge und die Zeit, welche sie für die Durchsicht meiner Präparate aufgewandt haben.

Sämtliche Figuren sind mit dem Abbe schen Zeichenapuarat von I)r. Wahrlich, welchem ich dafür meinen grossen Dank sage, ausgeführt. Die liguren 1, 5, 6, 8, 9, 10 bei Zeiss Oc 12, ()bj. $1,5 \mathrm{~mm}$ homog. Immersion; Hig. 3 bei Yeiss Oc 8 , Obj. 1,5 mm homog. lmmersion; Hig. 7 bei Zeiss ()c. 3, ()bj. 1,5 $\mathrm{mm}$ homog. Immersion; Yig. 2 und $t$ bei Zeiss Oc. 12, (Obj $3 \mathrm{~mm}$ homog. limmersion.

\section{Das Peter Miiller'sche Impressionsverfahren}

Eine kritische Studie ïber die Arbeit von Beuttner, /ur Frage der Einleitung der künstlichen Frühgeburt bei Beckenenge. Archiı für Gynaekologie, Band 18, Seite 269

Von

\section{F. AHL,FELI).}

Im iahre 1885 veröftientlichte P. Mijller seine Methode des Einplessens des Kopfes in das enge Becken zu diagnostischen Zwecken ${ }^{1}$ ). Besondere Vorteile, vermutete der Autor, würden aus diesem Verfahren der Sicherstellung der Zeit, wann die künstliche Frühgeburt aus\%uführen sei, erwachsen. Denn, so lange der Kopf noch in den Beckeneingang einpressbar sei, wiirde eine Friugreburt voraussichtlich noch Eifolg haben.

Als ich seiner Zeit den Vorschlag gelesen hatte, waren es \%uerst theoretische Bedenken, die mich an seinem Werte \%weifeln liessen, als ich ihn dann erprobte, kamen Bedenken nach der praktischen seite linzu und so habe ich, weil ich mit meinen anderen Methoden mindestens keine schlechteren Resultate und, wie icl, jetzt aus der Beuttner'schen Arbeit ersehe, bessere Resultate als P. M illler habe, iln später nicht wieder zur Anwendung gebracht.

Die erste Frage, die ich mir nach der Lecture des II ïller'. schen Vorschlags rorlegte, war: Wie ist es denn nun. wenn man den Kopf nicht mehr in das Becken einpressen kann? Ist dann die Ausfïhrung der künstlichen Frühgeburt erfolglos und daher contraindiciert? Nach meinen Eirfahrungen musste ich mir antworten: Keineswegs; denn ich hatte ja oft genug Geburten beobachtet, wo es trot\% der ausgiebigsten Anwendung der austreibenden Kräfte denselben nicht gelang, den Kopf als vorangehenden Kopf

1) Vortrag in der gynack. Section der Nacuriorscherversamnllung zu Strass. burg, 19. Sept. 1885. - Volkmann's Vortrïge, 3. Band, No. 264, S. 1887. 